\section{Myelodysplastic syndromes: from conductiong clinical trials of novel therapies to evaluating real-life effectiveness of exisiting therapies}

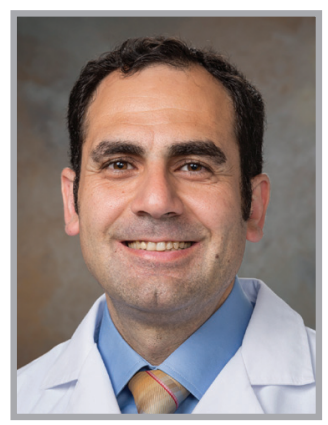

Amer M Zeidan* speaks to Laura Dormer, Editorial Director: Amer Zeidan is Assistant Professor of Medicine (Hematology) at Yale Cancer Center. He completed a hematology/oncology fellowship and a clinical research fellowship in myelodysplastic syndromes (MDS) at Johns Hopkins University where he also earned a Master of Health Science degree in Clinical Investigation. He received his MBBS degree from the Faculty of Medicine, University of Jordan (Amman, Jordan), before completing his internal medicine residency at Rochester General Hospital (USA). His clinical interest is in the management of hematologic malignancies. The focus of his clinical/translational research is the development of novel therapies for MDS and myeloid hematologic malignancies. The second area of research interest for him is effectiveness and outcomes research in hematologic malignancies, especially MDS.

\section{Q Could you briefly summarize your career path, \& how you came to specialize in} hematologic oncology?

I have always wanted to be a physician since I was a child. I was lucky enough to get accepted in one of the two medical schools in Jordan at the time; University of Jordan. In the fourth year of studying medicine, I decided to become a blood cancer doctor and researcher. That decision was largely related to a young child with an advanced cancer whom I cared for during my first clinical rotation and which turned out to be a life-changing experience. I did not want to just give chemotherapy and see patients, I wanted to be able to investigate novel drugs and offer new hope for the patients. I knew at the same time I wanted to complete my advanced training in the USA as it had all the resources I needed to become a successful translational/clinical researcher. After completing medical school and while completing the necessary exams to move to the USA, I trained in the King Hussein Cancer Center in Jordan for a year further solidifying my career decision. Thereafter I joined Rochester General Hospital Internal Medicine program and took my first steps in learning how to conduct clinical research and critically appraise the medical literature. The next step in my training was joining the hematology/oncology fellowship program at Johns Hopkins University, which also included significant research time followed by a dedicated year researching myelodysplastic syndromes (MDS). During that year I also completed a Master of Science program in Clinical Investigation at Bloomberg School of Public Health in Johns Hopkins University. Last year, I joined the Department of Internal Medicine at Yale University as an Assistant Professor in Medicine in the section of hematology.

*Section of Hematology, Department of Internal Medicine, Yale University, New Haven, CT 06520, USA; amer.zeidan@yale.edu

\section{KEYWORDS}

- effectiveness research

- hypomethylating agents

- lenalidomide $\bullet$ myelodysplastic syndromes $\bullet$ real-life analysis 
Q Are there any individuals you have worked with who have particularly influenced the path your career has taken?

I have been blessed to train and work with many excellent clinicians and top-notch researchers throughout my training and career. Among the people that influenced my career path and supported me at important junctures in my career the most were Dr Paul Bernstein, Dr Ronald Sham and Dr Balazs Zsenits at Rochester General Hospital, and Dr Judith Karp, Dr B Douglas Smith, Dr Michael Streiff and Dr Javier Bolanos-Meade at Johns Hopkins University. Dr Rami Komorkji from Moffitt Cancer Center also provided me with valuable guidance, collaborated with me on mutiple research projects, and has been a role model for me. My first mentor in Rochester was Dr Peter Kouides who introduced me to clinical research and helped me write my first paper and first study protocol, and who has continued to provide me with support and guidance throughout my career. Dr Meir Wetzler was my second mentor and helped me focus my research interests on leukemias and translational aspects of research and continued to provide me with honest advice and ongoing mentorship until his unexpected passing earlier this year, which was a very painful experience for me. Dr Amy Davidoff introduced me to outcomes and effectiveness research and continues to provide me with priceless mentorship. Last and definitely not least, Dr Steven Gore, a world-renowned scientist and researcher in MDS, introduced me in the second year of fellowship to clinical and translational research in MDS, which has since become my passion. Steve has helped me conduct multiple projects that have been presented at prestigious national and international meetings, write important research papers and obtain noticable unding and awards. Steve continues to be my mentor at Yale University where we work together on promising translational/clinical research, and effectiveness and outcomes research in myeloid hematologic oncology, especially MDS.

Q You are currently Assistant Professor of
Medicine (Hematology) at the Yale Cancer
Center; how do you divide your time between
clinical \& research work?
At Yale we have a strong clinical and research
team for myeloid malignancies that is led by
experienced translational/clinical researchers (Dr

Thomas Prebet and Dr Nikolai Podoltsev) and labaratory-based translational physician scinetists (Dr Stephanie Halene and Dr Manoj Pillai) in additon to Dr Gore and myself. We are well supported by an active clinical research office and state-of-the-art labaratorties and core facilities that conduct cutting edge science in addition to an enlarging tissue bank and a clinical database. Currently at Yale I attend on the oncologic malignancy inpatient service for 3 months of the year and have three half-day clinics that are dedicated to myeloid malignancies, especially MDS. The rest of my time is dedicated to research activities. As any clinical investigator, the clinical activities feed directly into my research in terms of enrollment into clinical trials and observational studies in addition to obtaining tissue and blood samples for labaratory research with my collaborators.

Q One of your areas of research interest is outcomes research in MDS; what is your research focusing on at present?

Effectiveness and outcomes research is very important because it allows assessment of patient outcomes at the population level in contrast to the clinical trials that involve highly selected groups of patients that are enrolled with strict eligibility criteria in a very controlled environment. This type of research is vital to make sure that the efficacy of drugs demonstrated in clinical trials is actually being translated into clinical effectiveness in the real-life setting. I am lucky to work with a very experienced multidisciplinary research team in the Cancer Outcomes, Public Policy and Effectiveness Research (COPPER) Center at Yale University composed of experts in large database analyses, epidemiologic studies and health economics (Dr Amy Davidoff, Dr Xiaomei Ma and Dr Cary Gross), experts in programing and statistical analysis, in addition to Dr Steven Gore. These resources make us uniquely positioned to address very important clinical questions regarding the effectiveness and safety of the different drugs used for treatment of patients with MDS at the population level (e.g., lenalidomide, azacitidine and decitabine) in addition to cost-effectiveness. These studies can have important implications as they inform doctors and patients with real-life effectiveness data regarding the drugs they might consider using.

Q You have used clinical databases \& insurance claims data in your outcomes 
research; what are the specific challenges you face using these kinds of data?

Our outcomes research has focused around the use of data from the National Cancer Institute's Surveillance, Epidemiology, and End Results (SEER) program, which is linked to Medicare enrollment and claims data. Medicare is the primary insurance for patients who are 65 years or older in the USA, and the fact that $80 \%$ of patients in the USA diagnosed with MDS are $\geq 65$-year-old renders MDS particularly suited for analysis using administrative data from the SEER-Medicare program. The main challenge in using this type of dataset is the lack of availability of important clinical and pathologic parameters such as the karyotype and blast percentage, but those aspects are compensated for by the ability to study tens of thousands of patients with a rare cancer such as MDS, a number that is not currently possible to research in any other patient registry.

\section{Q What do you hope to achieve with your research in the next few years?}

My translational/clinical research in MDS focuses on finding novel active therapies for MDS especially using immunologic approaches such as immune-checkpoint inhibitors and epigenetic approaches. No new drug has been approved for use in MDS since 2006 and we hope that will change soon by the collective contributions of MDS researchers around the world. My outcomes research is focusing on understanding the real-life effectiveness of the two most commonly used drugs for higher risk MDS (azacitidine and decitabine), including questions related to their comparative clinical and cost-effectiveness, outcomes achieved with different administration schedules and routes, and effects of sequential therapy with the goal of identifying the drug and approaches associated with best patient outcomes in the real-life setting.
Q What do you think will be the key developments in the management of MDS in the next $5-10$ years?

MDS research over the next few years will bring us better understanding of the molecular pathogenesis of the disease and how to use this information to rationally design effective therapies to improve patient outcomes. We also hope to understand how to use these novel biomarkers to identify patients with high likelihood of benefit from hypomethylating agents or allogeneic bone marrow transplants to select such patients for upfront clinical trials. Other important unmet clinical need in MDS include the discovery or development of drugs that are efficacious in the posthypomethylating agent failure setting, which is associated with very poor survival. The results of hypomethylating agents could be improved in the upfront setting by combination-based approaches (although to date trial data have been largely disappointing in this regard) or by designing more active epigenetic agents. Improving the applicability of the only potentially curative treatment modality for MDS, allogeneic hematopoietic cell transplantation, by decreasing the toxicity of the procedure and increasing graft sources is also an important research direction.

\section{Disclaimer}

The opinions expressed in this interview are those of the interviewee and do not necessarily reflect the views of Future Medicine Ltd.

Financial \& competing interests disclosure

AM Zeidan has no relevant affiliations or financial involvement with any organization or entity with a financial interest in or financial conflict with the subject matter or materials discussed in the manuscript. This includes employment, consultancies, honoraria, stock ownership or options, expert testimony, grants or patents received or pending, or royalties.

No writing assistance was utilized in the production of this manuscript. 